\title{
THE ABSORPTION AND REFLECTION OF CALCITE AND ARAGONITE FOR INFRA-RED RAYS AS DEPENDENT UPON THE PLANE OF POLARIZATION. ${ }^{1}$
}

By R. E. Nyswander.

$\mathrm{T}^{\mathrm{H}}$ $\mathrm{HE}$ structure and vibration periods of molecular aggregations would naturally first be sought in compounds of simplest composition. Certain groups of atoms having particular free periods by themselves may lose these periods when related in a complicated manner. Previous investigations in the infra-red of absorption and reflection spectra have shown that each of the simple compounds of the same acid radical, viz., nitrates, sulphates and carbonates have characteristic bands in the same regions of the spectrum. The carbonates, as demonstrated by Morse, ${ }^{2}$ show reflection maxima in the infra-red, the positions of which vary with the atomic weight of the base in an approximately straight line relation.

The most direct method of attacking the question of molecular structure is through the behavior of crystalline media, since the vibrations are limited to definite directions in the crystal. Little has been done in this direction, in part for the want of a suitable polarizer for long waves, and largely because of the loss of energy by polarization. As regards selective reflection as dependent upon the plane of polarization, the field is new.

That absorption in doubly refracting media is dependent upon the direction of vibration of the incident energy has been demonstrated conclusively by the work of Merritt ${ }^{3}$ and of Königsberger. ${ }^{4}$ In the case of calcite the independence of the absorption for the

${ }^{1}$ Presented by title at the Washington meeting of the American Physical Society, April 24-25, 1908. Abstracted in the Physical Review, 26, 539, 1908.

2 Astrophys. Jour., I1, 225, 1907.

3 Phys. Rev., 2, 424, 1895.

Ann. der Phys., 61, 687, 1897. 
[VoL. XXVIII.

ordinary and extraordinary rays was especially marked. The work was not carried beyond $5.5 \mu$, due to the loss of energy by polarization.

The identity of composition of calcite and aragonite together with the differences in crystallographic structure of the two substances, suggested them as worthy of further investigation. The large differences in the indices of refraction for two of the principal directions of vibration of both calcite and aragonite, and the simplicity of composition, would hint that large differences in absorption and reflection might be expected.

The work of this investigation consisted of two parts, dealing respectively with the absorption and reflection spectra for the principal directions of vibration of calcite and aragonite.

Description of Apparatus.

A diagram of the apparatus as arranged is shown in Fig. I.

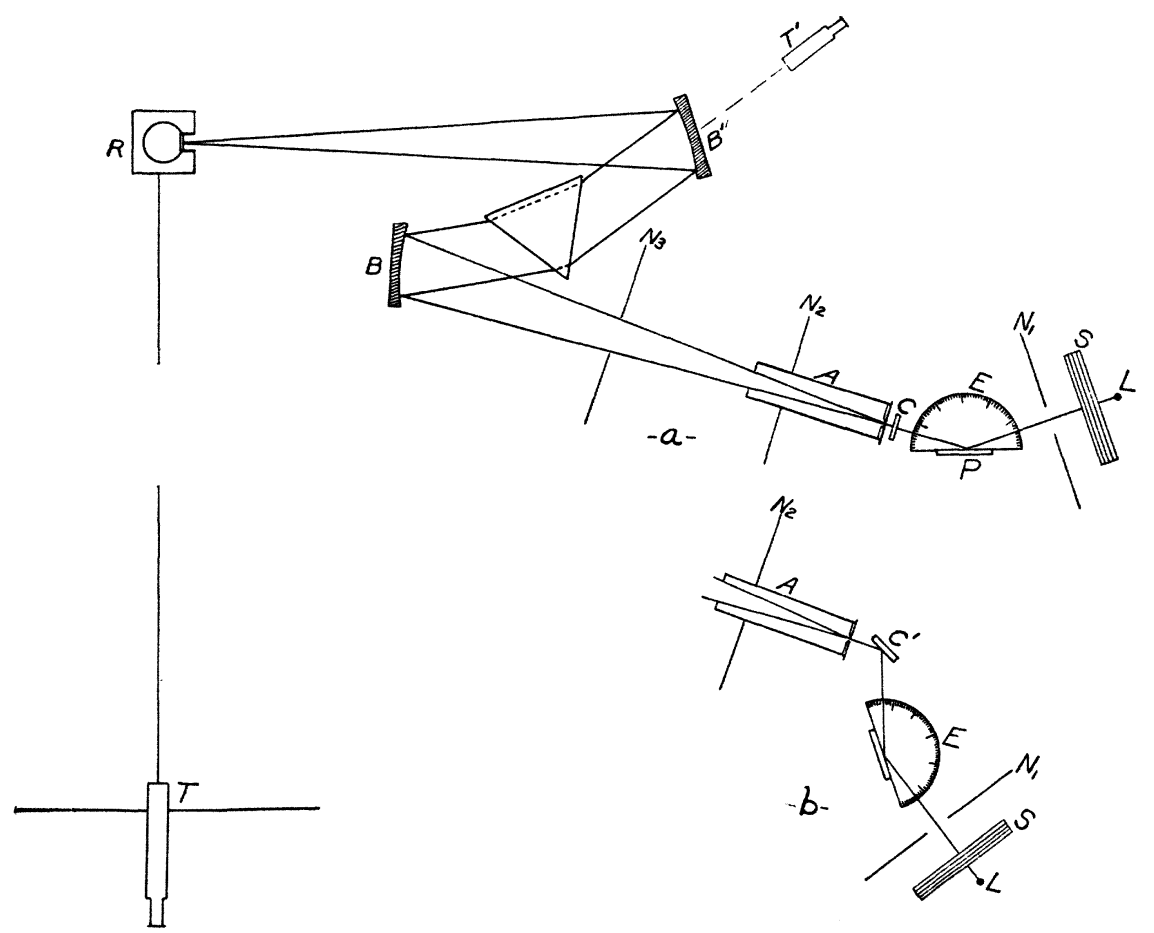

Fig. 1. 
For the transmission spectra the collimating $\operatorname{arm} A$ of the spectrometer carried an attachment as indicated in $a$; for the reflection curves, this attachment was modified as shown in $b$.

Absorption. - Rays from a Nernst heater $L$ fell upon the polarizer $P$ from which they were reflected into the slit of the spectrometer, and after reflection from the concave minor $B$ passed through the prism and were brought to a focus upon the radiometer $R$ by the second concave minor $B^{\prime}$. Deflections were produced by raising the shutter $S$. The crystal section, whose transmission spectrum was desired, was placed at $C$. The crystal was mounted on a simple mechanism permitting it to be thrown in the path of the rays, always in the same position.

Reflection. - The same polarizing device used for the absorption work, was also used for the reflection curves as shown in $b$. Rays from the polarizer $P$ were reflected to $C^{\prime}$, and from there to the spectrometer slit. The device at $C^{\prime}$ carried the crystal section attached to one of two arms, which turned about a common center. To the other arm was fastened a silver mirror for comparison. Either arm, when turned into position, dropped into a notch, pressing the reflecting surface against a fixed guide, which brought it to the same position for each observation.

The arm carrying the source $L$ could be turned about the center of the graduated arc $E$ as axis. The polarizer was attached rigidly to this graduated arc, the combination being free to turn about its center.

The scale $T$ was placed $\mathrm{I} 20 \mathrm{~cm}$. from the radiometer.

Spectrometer. - The reflection spectrometer had a focal length of $35 \mathrm{~cm}$. The method of constant angle of emergence was employed, so that the source and likewise the devices for polarization, absorption and reflection were attached rigidly to the collimating arm. These attachments could produce no strain in the instrument since the whole weighed less than one-half kilogram. The rocksalt prism was the same one used by Coblent ${ }^{1}$ during his investigations in this laboratory. As no changes in the prism faces had been made since that time, his calibration curve was used by the author also. The prism faces were $7 \mathrm{~cm}$. square, and the refracting

\footnotetext{
${ }^{1}$ Investigations of Infra-red Spectra, 16.
} 
angle $59^{\circ} 57^{\prime} 43^{\prime \prime}$. A circular plate of glass somewhat larger than the dimensions of the prism was cemented on the prism table. Upon this plate the prism was accurately mounted. A small beaker containing phosphorus pentoxide was placed on the prism to absorb moisture. A bell-jar attached to a weighted cord, passing over a pulley above, could be lowered over the prism without jar to the apparatus and fit tightly upon the glass plate.

The angle of emergence was the angle of minimum deviation for sodium light. This angle was determined by the use of a telescope, Fig. I, $T^{\prime}$, set up in line of the deviated rays from the prism. The collimating arm was first clamped at a convenient point on the spectrometer circle. The image of the slit was focused on the graduated scale of the eyepiece and the prism table adjusted to the angle of minimum deviation. The other arm of the spectrometer was then turned in position and adjusted until the image fell upon the radiometer slit. The settings were further checked by the position of the $\mathrm{CO}_{2}$ band, from a bunsen burner, which falls sharply on $4.40 \mu$.

Radiometer. - A Nichols radiometer used by previous observers in this laboratory, was at hand. Several improvements, however, were added. The sensitiveness of the radiometer was determined in a former investigation. With a candle and scale at one meter distance a deflection of $\mathrm{I} 2.5$ centimeters per square millimeter of exposed vane area was produced.

Vanes. - The superiority of platinum black as an absorbent of radiant energy has long been recognized. To obtain a uniform electrolytic deposit without the added weight of metallic vanes, the following method was successful. Thin sheets of mica were platinized on one side with platinum chloride. The $\mathrm{PtCl}_{4}$ was applied to the surface with a soft brush and allowed to dry. The mica was then heated over a metal plate until a thin layer of platinum appeared. This process was repeated several times until a uniform conducting layer was formed. In order that the platinum stick to the mica, it is essential that the surface of the mica be roughed with medium emery paper before the $\mathrm{PtCl}_{4}$ is applied. Sheets of mica thus prepared were coated with platinum black after the electrolytic process of Kurlbaum. ${ }^{1}$ The vanes were $\mathrm{I} \times$ I I mm. and the sus- 
pended system weighed $9 \mathrm{mg}$. The mirror was plane, about $2 \times 3 \mathrm{~mm}$. The surfaces were ground, and the image was perfect. The fiber which was about $4.7 \mathrm{~cm}$. in length, was attached to the bottom of a stirrup carrying a small bar magnet one centimeter long. This stirrup was attached with a short silk fiber to a support at the top of the radiometer chamber. Around the neck, leading from the radiometer, was a movable collar carrying a control magnet, which had first been bent into shape so its poles would lie in the plane of the suspended magnet. This device offered most excellent control of the suspended system. A double rock-salt window was set back just in front of the vanes. The slit was set back to the front surface of the window, so that the loss in definition of the image between the slit and the vanes was reduced to a minimum. The device carrying the slit rested in two guides on the front of the radiometer, allowing lateral adjustment. The width of both the radiometer and spectrometer slits was equal. For all observations from $\mathrm{I} .7 \mu$ to $9.8 \mu$ this width was $.3 \mathrm{~mm}$., equivalent to $3^{\prime}$ of arc on the spectrometer circle. For observations beyond $9.8 \mu$, the slits were widened to $.55 \mathrm{~mm}$., or $5.5^{\prime}$ of arc. The time for a single deflection varied from .5 to I. 5 minutes when the radiometer was worked at its greatest sensibility. For observations in the more intense part of the spectrum the pressure was lowered until the radiometer acted ballistically. The time of deflection was thus reduced to 20 seconds. Within the tube leading from the radiometer was placed absorbent cotton mixed through with gold foil to absorb mercury vapor from the pump; no trouble from electrification of the vanes was experienced (see Coblentz). ${ }^{1}$

Source. - A Nernst heater operated on a well regulated I Io volt direct current circuit furnished the source of energy. The heater was run somewhat above its rated voltage and served as a very satisfactory source.

Polarizer. - Selenium, as shown by Pfund, ${ }^{2}$ has the properties necessary for a good reflection polarizer for long waves. Pfund's investigations were carried to I $3 \mu$; however, it was found in the present investigation to reflect uniformly beyond I $5 \mu$. A reflect-

${ }^{1}$ Investigations of Infra-red Spectra, Appendix III., I 24.

${ }^{2}$ Astrophys. Journ., I, 23, 1906. 
ing polarizer was made after this method, and polarization was complete. The angle of polarization was determined experimentally with a Nicol's prism. This angle, as measured on the device of Fig. I, was $71.5^{\circ}$.

Crystal Sections. - Polished sections cut by Zeiss from clear crystals of calcite and aragonite, and containing the principal directions of vibration, were employed. The section of calcite was I $\mathrm{mm}$. thick, and cut parallel to the optic axis. The two aragonite sections were cut parallel to the $a b$ and $a c$ axes respectively. Large untwinned crystals of aragonite are difficult to obtain. The crystals examined were of the Bohemian type and of clear quality. The thickness of these plates was $2 \mathrm{~mm}$., and the smaller one was about $\mathrm{I} \mathrm{cm}$. square. For a more definite location of the calcite bands of absorption than could be gained from the above section on account of its thickness, a thin section of calcite, . $14 \mathrm{~mm}$. in thickness, cut $70^{\circ}$ to the optic axis, was also examined. The curves of this section serve well to show the great differences of absorption as dependent upon thickness.

A shutter consisting of five parallel plates of tin rendered radiation from the source through the shutter quite impossible. The plates of tin were placed about $.5 \mathrm{~cm}$. apart, permitting free circulation of air between them. The shutter was fastened from the ceiling and was operated with a cord by the observer. Observations were made in a small basement room completely surrounded by other rooms, and for this reason the temperature changes were very small. The spectrometer and radiometer were mounted on a table which itself stood on marble blocks imbedded in saw-dust, in boxes resting on the concrete floor. Very little trouble was experienced from tremors. Every precaution was taken to protect the apparatus from outside radiations. Both spectrometer and radiometer were completely covered. As a further protection to the spectrometer from direct radiations of the source, opaque screens were placed at $N_{1}, N_{2}, N_{3}$. The source, polarizer, and slit were also screened from external radiations. The radiometer was kept connected with the mercury pump, but the stop-cock was always closed except during adjustments for sensibility, to prevent the diffusion of mercury vapor to the radiometer. All connecting joints were mercury sealed and little trouble with leaks was experienced. 


\section{Method of ObServation.}

The same crystal sections were used for both the absorption and reflection curves. The fraction or percentage of transmission was the ratio of the deflection when the crystal was placed in the path of the polarized rays to the deflection when it was removed. Likewise, the reflection percentage was the ratio of the reflection from the crystal to that of silver assumed 100 per cent.

The plane of the polarizer was placed in a vertical position. A vertical strip of the reflecting surface about $\mathrm{I} \mathrm{cm}$. wide was used, the remaining part of the surface being screened with black paper. According to the theory of Fresnel, the direction of vibration of the polarized energy would also be in a vertical plane. The particular crystal direction to be examined was placed parallel to the direction of vibration of the incident energy.

The planes of polarization of these substances, as determined by the crystallographic axes, may be further defined as follows: The axis $a$ is the direction of least elasticity and greatest index of refraction, and a normal to the plane of the optic axes. The $b$ direction is the obtuse bisectrix of the crystal, and the direction of medium elasticity and medium index of refraction. The $c$ direction is the acute bisectrix, and the line of greatest elasticity and least index of refraction. For calcite the $c$ axis is parallel to the optic axis. The $a$ and $b$ axes are equal.

All observations were repeated one or more times in parts of the spectrum where the energy was large, and usually six to eight times when the deflections were small. In each case the zero was determined by the mean of two readings, one before, and one after each deflection. With the crystal in position, the zero was noted at the instant the shutter was raised. When the mirror came to rest, the deflection was read and the shutter lowered; the crystal was turned out of the path of the energy and a second zero read, and at the same time a second exposure made after which the zero was again read. The middle zero was used in determining the mean zero of the two deflections. Before starting the observations, it was necessary to remain in the room at least a half hour until the temperature conditions became constant. With the excellent definition of the image of the scale, deflections were readily esti- 
mated to tenths of a millimeter. Recorded results are the means of all observations.

Due to deterioration of the silvered surfaces, two comparison mirrors were used for the reflection curves, viz., from I.7 $\mu$ to $9.8 \mu$ and $9.8 \mu$ to $14.5 \mu$ respectively. In each case special care was taken to secure the best polished surface.

\section{RESULTS.}

In the present investigation the curves of absorption and reflection for the principal directions of vibration of calcite and aragonite have been carried beyond the absorption and reflection bands at 14.2 $\mu$. All curves have been drawn to the same scale in Figs. 2 to

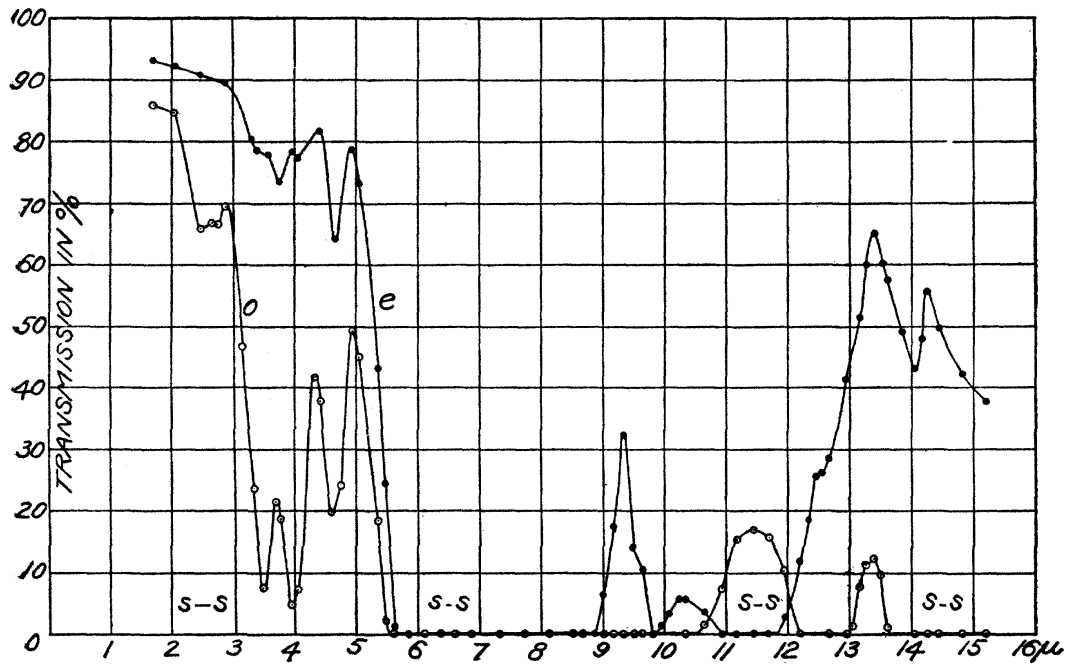

Fig. 2. Transmission curves for calcite $\left(\mathrm{CaCO}_{3}\right)$, thickness $1.0 \mathrm{~mm}$. o, ordinary ray ; $e$, extraordinary ray ; $s-s$, width of slit.

4 , and 7 to 9 . The reflection bands have been drawn to twice this scale of wave-length in Figs. 5 and 6, and 10 to I 3.

Calcite. Transmission. - Observations were first made on the section of I mm. thickness. The ordinary ray (Fig. 2,o) shows a shallow absorption band at $2.46 \mu$. Strong bands are present at $3.46 \mu, 3.96 \mu$ and $4.58 \mu$. The section becomes opaque at $5.54 \mu$ and continues to $10.52 \mu$. The ray is again completely absorbed from $\mathrm{I} 2.2 \mu$ to $\mathrm{I} 3.0 \mu$, and beyond $\mathrm{I} 3.63 \mu$. 
The extraordinary ray (Fig. $2, e$ ) indicates absorption bands at $3.74 \mu$ and $4.65 \mu$ and becomes opaque at 5.6I $\mu$, continuing opaque to $8.83 \mu$. Another band falls sharply at $9.80 \mu$. The ray is also completely absorbed between $10.9 \mu$ and II.8 $\mu$. The percentage transmission rises to 65.0 per cent. at $\mathrm{I} 3.40 \mu$. A small absorption band falls sharply at $14.06 \mu$.

These results were compared with the work of Coblentz ${ }^{1}$ who determined the transmission spectrum of calcite with unpolarized rays from I $\mu$ to $14.2 \mu$ with a crystal section . I $4 \mathrm{~mm}$. in thickness cut $70^{\circ}$ to the optic axis. The curves for the two sections were so different that little relation could be established between them. Further, in comparing this curve with the curves of Merritt there seemed to be greater discrepancies. The specimen of Coblentz being cut $70^{\circ}$ to the optic axis contains a predominance of the ordinary vibration, and its transmission curve should agree the closer with that of the ordinary ray, but the reverse relation was quite evident. An exactly similar section cut $70^{\circ}$ to the axis was obtained and its transmission spectra plotted for the two planes of

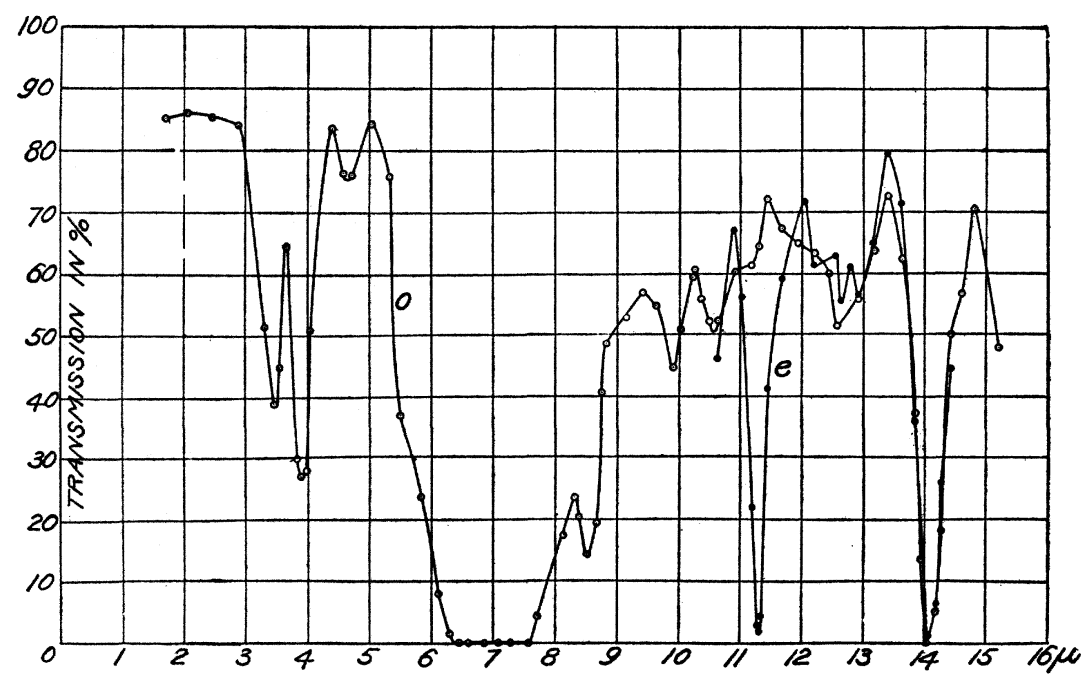

Fig. 3. Transmission curves for calcite, thickness $0.14 \mathrm{~mm}$. $o$, ordinary ray ; $e$, extra. ordinary ray.

polarization. The results from this section (Fig. 3) showed that the work of Coblentz was correct to $14 \mu$, and the differences exist-

${ }^{1}$ Investigations of Infra-red Spectra, Part III., 7. 
ing for the two sections in question were accounted for entirely by difference in thickness. Likewise the seeming discrepancy with Merritt's work was readily explainable on this ground. The width, as also the depth of absorption bands increases with the thickness of the section so that for thin sections certain small bands completely disappear. The curve of the ordinary ray (Fig. 3,o) represents the pure absorption of this ray, but for the extraordinary ray (Fig. 3,e) the results are slightly masked by the behavior of the ordinary ray. The principal bands of the ordinary ray between $2 \mu$ and $6 \mu$ for both sections agree. The broad band of complete absorption in the region of $7 \mu$ lies within the limits $6.36 \mu$ to $7.60 \mu$ for the thin section. The bands at $8.50 \mu, 9.92 \mu$ and $10.57 \mu$ are not brought out by the thick section. These curves show conclusively that the absorption band at II. $3 \mu$ is due entirely to the absorption of the extraordinary ray. There is no trace of an absorption band in this region for the ordinary ray. At $\mathrm{I} 2.66 \mu \mathrm{a}$ band is present in both

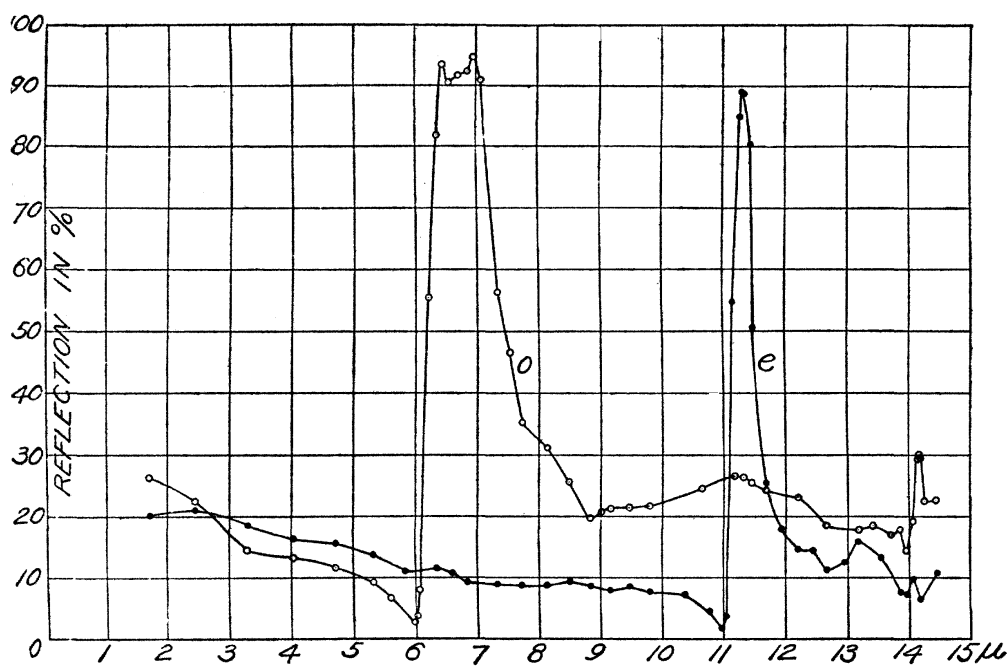

Fig. 4. Reflection from calcite. $o$, ordinary ray; $e$, extraordinary ray.

rays. The point of maximum transmission occurs at $\mathrm{I} 3.4 \mu$ for both rays and the transmission for the two is not very different. But the curves of Fig. 2 serve well to show that the coefficient of absorption in this region is much greater for the ordinary ray than for the extraordinary ray. The ordinary ray is completely absorbed 
at $14.06 \mu$ while the extraordinary ray shows only a shallow band in this region for the thick section. Due to the component which the ordinary ray exerts in the thin section, the extraordinary ray will show the band at $14.06 \mu$ much exaggerated in depth. The positions of the absorption bands of calcite are given by Kayser ${ }^{1}$ as follows : $6.69 \mu, \mathrm{II} .4 \mathrm{I} \mu, \mathrm{I} 4.4 \mu$ and $29.4 \mu$. In the present investigation, if the center of the first band be taken as $6.7 \mathrm{I} \mu$, the variations from the above values are $+.02 \mu,-$ I I $\mu$ and $-.3 \mu$ respectively.

Reflection. - The reflection curves for calcite have relations similar to the absoption curves. The first reflection band occurs in the ordinary ray (Fig. $4, o$ ). It is broad and complex, with two principal and nearly equal maxima at $6.46 \mu$ and $6.96 \mu$, and indication of another maxima at $6.70 \mu$. This band is no doubt more complex than indicated in this curve. Coblentz ${ }^{2}$ with unpolarized energy found a complex reflection band for calcite with maxima at $6.5 \mu$ and $6.6 \mu$, and a complex maximum in the region of $7 \mu$. The absence of any absorption band at II.3 $\mu$ in the transmission curve of the ordinary ray is likewise brought out by the absence of a reflection band in this region for the ordinary ray.

For the extraordinary ray (Fig. $4, e$ ) which showed complete absorption in the regions $6.7 \mu$ and II $3 \mu$ for the thick section, there undoubtedly would have been complete absorption in the latter region by the thin section, except for the direction in which the crystal was cut. This ray shows no trace of a reflection band at $6.7 \mu$, but a very strong band, reflecting $89 . \mathrm{I}$ per cent. of the incident energy at II.3O $\mu$. The position of this band from the transmission curve is I I.28 $\mu$. This small difference might readily be explainable again by the impurity of the extraordinary curve. As shown in Fig. 3, the ordinary curve is changing very rapidly in this region and in a manner to shift the minimum toward the shorter wave-lengths. Both curves exhibit reflection bands beyond I $4 \mu$. For the extraordinary ray in which absorption was not complete, the change in reflection is also small, and coincides in position with the absorption band. The ordinary ray is com-

${ }^{1}$ Handbuch der Spectroscopie, Vol. IV., 502.

2 Supplementary Investigations of Infra-red Spectra, Part V., 23. 
[VoL. XXVIII.

pletely absorbed at $\mathrm{I} 4.06 \mu$ but its reflection band is shifted to 14. I $7 \mu$. The importance of exact setting of the crystal is brought out by the broken curve $a$, Fig. 6 . This band, since it was not a typical reflection band, led to a question of error in setting the crystal, or incompleteness of polarization. The crystal was reset with
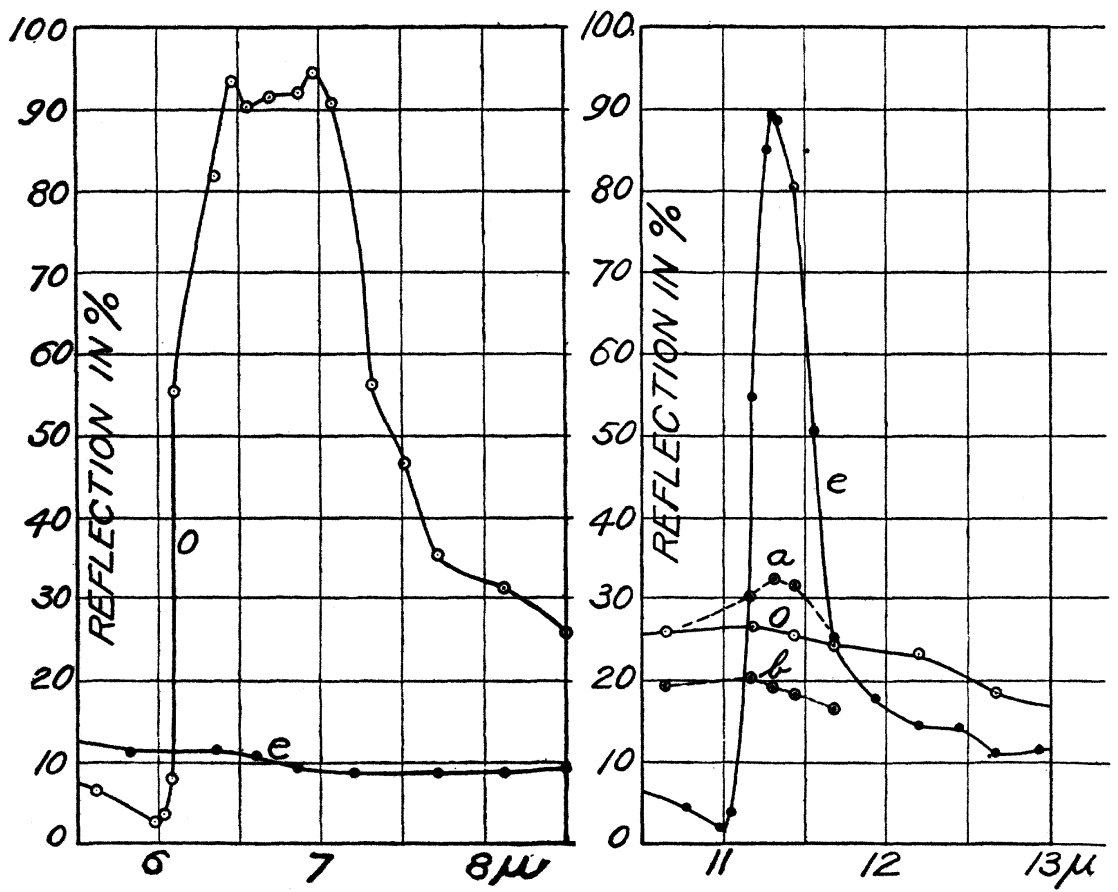

Figs. 5-6. Reflection from calcite. o, ordinary ray ; e, extraordinary ray.

greater exactness and this region reëxamined. The full curve $o$ was obtained. To check this point further another section cut perpendicular to the optic axis was investigated through this region. In this curve there could be little possibility of the extraordinary ray exerting an influence, and for this crystal the broken curve $b$, Fig. 6, was obtained, checking the curve $o$.

Aragonite. Transmission. - When the direction of vibration of the incident energy is parallel to the $a$ axis (Fig. $7, a$ ) the curve is not very different from the ordinary curve for calcite. The first band appears as $2.46 \mu$, another at $3.30 \mu$, and then a band of zero 
transmission at $4.04 \mu$. At $5.54 \mu$ there is complete absorption continuing to $9.50 \mu$. This direction transmits very little energy in the region $9.5 \mu$ to $\mathrm{I} 2.0 \mu$. At $\mathrm{I} 3.92 \mu$ the curve falls to zero and continues to the end of the observations.

The curve for the second direction of vibration (Fig. $7, b$ ), parallel to the $b$ axis, is somewhat similar to the transmission curve $a$, but

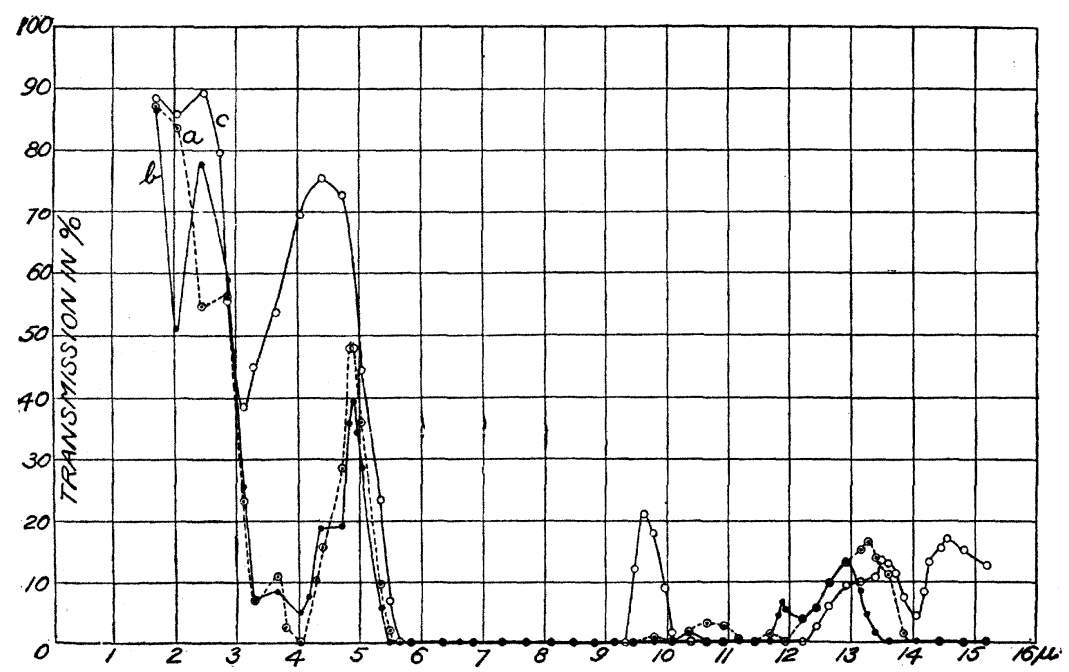

Fig. 7. Transmission curves for aragonite $\left(\mathrm{CaCO}_{3}\right)$, thickness $2.0 \mathrm{~mm}$. $a$, vibrations $\| a \perp b$ axis ; $b$, vibrations $\| b$ axis ; $c$, vibrations $\| c$ axis.

shows a shift toward the shorter wave-lengths of some of its maxima and minima. Absorption bands are present at $2.05 \mu, 3.30 \mu$ and $4.04 \mu$. The absorption is complete from $5.45 \mu$ to Io. Io $\mu$, and again between $10.6 \mu$ and I I.7 $\mu$. Another band appears at I $2.20 \mu$, and beyond I $3.5 \mu$ the transmission is zero.

When the incident vibrations are parallel to the $c$ axis (Fig. 5, $c$ ) the transmission curve resembles the extraordinary curve for calcite. This resemblance is especially close in the region $9 \mu$ to ${ }_{5} 5 \mu$ except that the transmission maxima and minima in the aragonite curve are shifted about $.25 \mu$ toward the longer waves in all cases except the band at $14.06 \mu$, for which there is exact coincidence. From I. $7 \mu$ to $5.5 \mu$ this curve shows a greater transmission of energy than curves $a$ and $b$. In this region there is a shallow band at $2.05 \mu$ and a deep band at $3.13 \mu$. Absorption is complete from 
$5.65 \mu$ to $9.32 \mu$. Again there is opacity from IO. I $3 \mu$ to $\mathrm{I} 2.26 \mu$. A sharp absorption band falls at $14.06 \mu$.

According to the theory of the ellipsoid of elasticity, the optical properties of a crystal are dependent upon the displacement of the particle in the direction of vibration, and independent of the ray direction through the medium. The two sections of aragonite contained four principal directions of vibration, two of which were parallel to the common axis $a$. As a further check upon the work in hand and to demonstrate that in a biaxial crystal absorption is dependent only upon the direction of vibration, the absorption curves for the directions $a, a$ in the two sections were obtained (Fig. 8). The

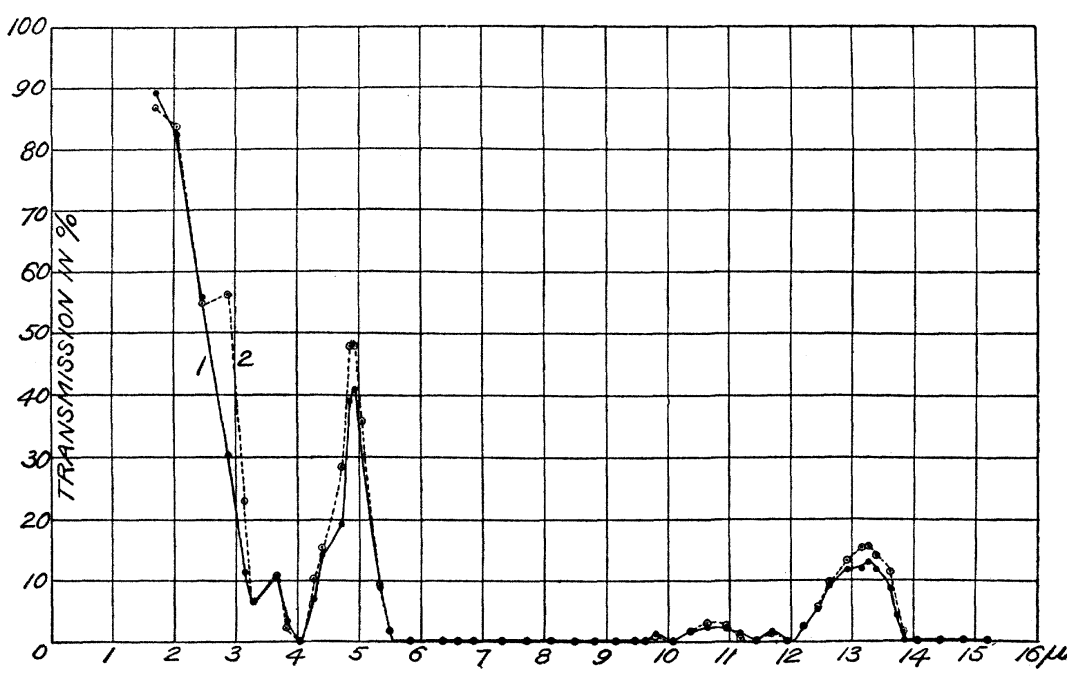

Fig. 8. Transmission curves for aragonite, thickness $2.0 \mathrm{~mm}$. I, vibrations $\| a \perp c$ axis ; 2, vibrations $\| a \quad b$ axis.

ray directions through the crystal in the two cases were normal to each other, but the directions of vibration parallel. The two curves show that the absorption characteristics are very similar. For curve $\mathrm{I}$, the ray direction was parallel to $b$, and parallel to $c$ for curve 2. The difference of note is the band at $2.46 \mu$ which occurs in curve 2 , but is entirely absent in I. In most parts the transmitting power of 2 is greater than $I$.

Reflection. - When the incident energy is parallel to the $a$ axis (Fig. 9, a) nearly Ioo per cent. of this energy is reflected at $6.65 \mu$. 
It is universally true that substances showing powerful selective absorption also exhibit correspondingly large reflecting powers in the same regions, yet few substances reflect so powerfully as this. Because of the sharpness and high reflecting power of this band, its

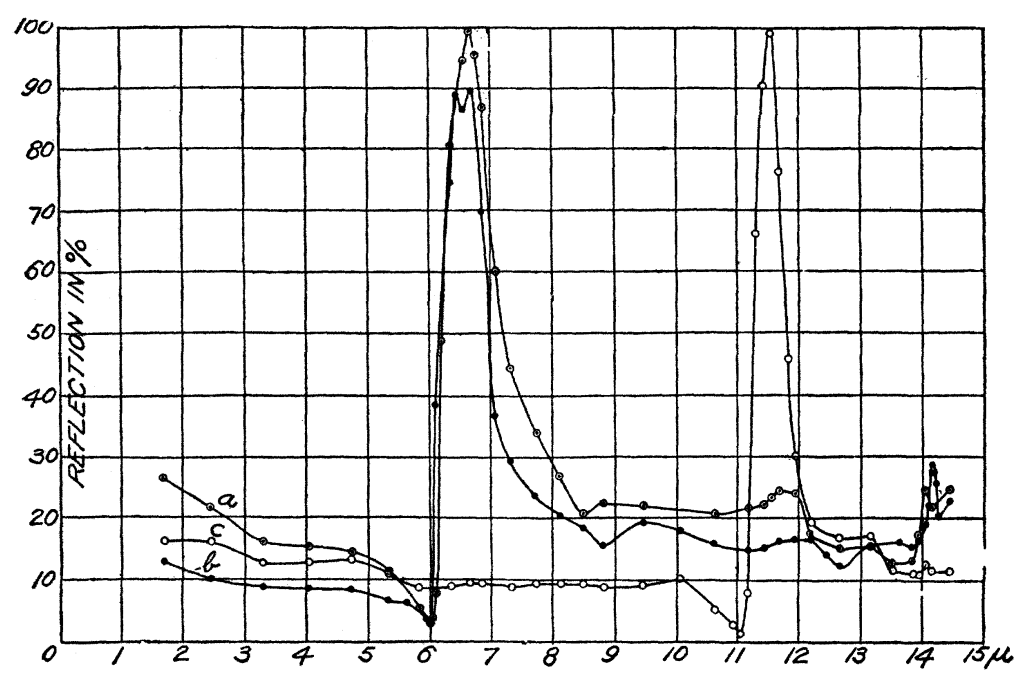

Fig. 9. Reflection from aragonite. $a$, vibrations $\| a \perp c$ axis; $b$, vibrations $\| b$ axis ; $c$, vibrations $\| c$ axis.

maximum must necessarily determine the position of the absorption maximum for this ray. No band is present at II.55 $\mu$ for this direction. Its second band falls at $14.06 \mu$ and through this region absorption was complete for the corresponding transmission curve.

In the second reflection curve (Fig. $9, b$ ) the direction of vibration is parallel to the $b$ axis. This curve has a complex band in the first region. The band has two sharp and nearly equal maxima which fall at $6.46 \mu$ and $6.70 \mu$ respectively. Coblentz, ${ }^{1}$ with unpolarized energy, found a complex reflection band for aragonite with two maxima at $6.53 \mu$ and $6.75 \mu$ respectively. The first maxima of the complex band of calcite occurs also at $6.46 \mu$. There is no band at $\mathrm{I} 1.55 \mu$, but at $\mathrm{I} 4 . \mathrm{I} 7 \mu$ a sharp band is present with a reflecting power of nearly 29 per cent. The transmission curve for this ray also shows opacity in this region. The position 
of this band agrees exactly with the corresponding band for the ordinary ray of calcite.

For the third curve the direction of vibration is parallel to the $c$ axis (Fig. 9, c). In the region $6 \mu$ to $7 \mu$ there is no trace of a
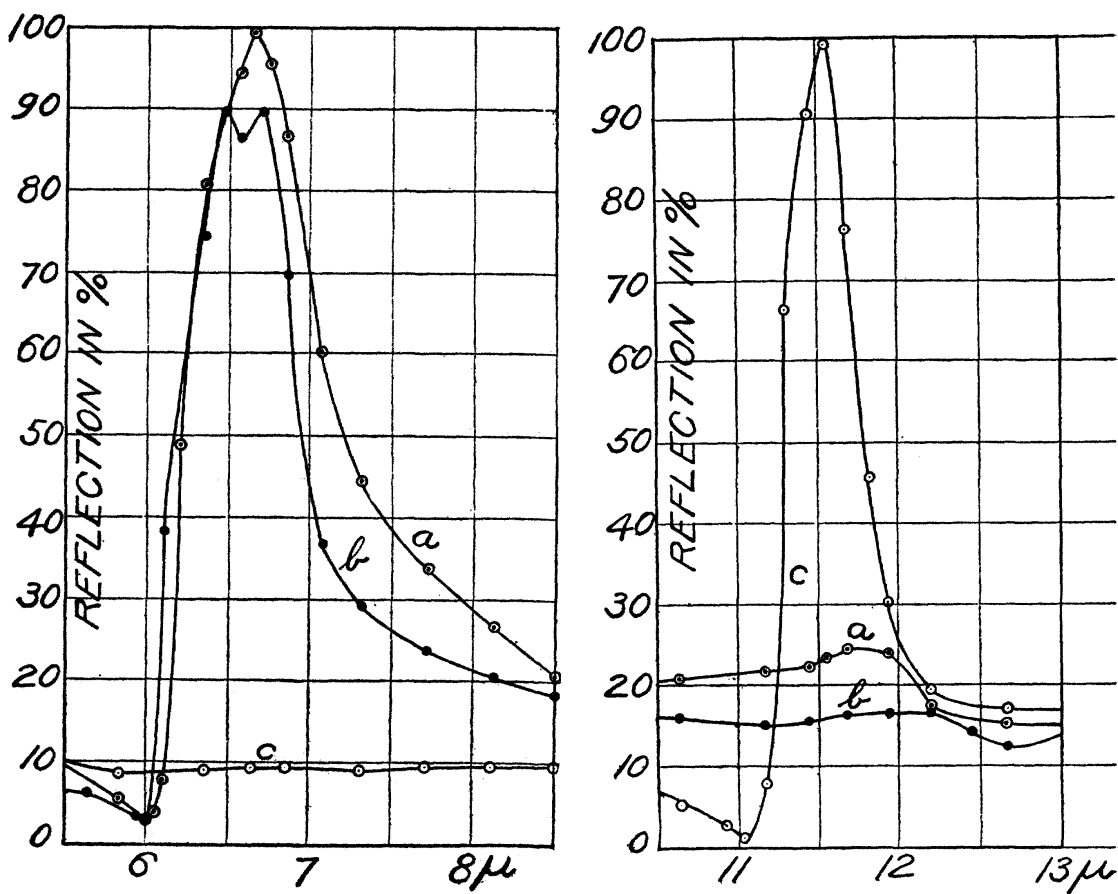

Fig. 10-11. Reflection from aragonite. $a$, vibrations $\| a$ axis; $b$, vibrations $\| b$ axis ; $c$, vibrations $\| c$ axis.

band, but in the second region there is a very strong band falling sharply at II.55 $\mu$, with a reflecting power of more than 99 per cent. This band like the first band of curve $a$, must determine the position of maximum absorption. At $14.06 \mu$ there is a small band which coincides in position and of about the same intensity as the band of the extraordinary ray of calcite. In fact the two curves are very similar except for the shift of the principal band which for calcite falls sharply at I I.30 $\mu$.

\section{SUMmaRY.}

The results of the foregoing investigation may be summarized as follows : 
I. The absorption and reflection curves of calcite and aragonite, as dependent upon the direction of vibration between I.7 $\mu$ and I $5 \mu$, show three regions of characteristic bands at $6.6 \mu, \mathrm{I}$ I. $5 \mu$ and $\mathrm{I} 4$. I $\mu$ respectively.

2. For calcite the reflection band in the first region is complex with two principal maxima at $6.46 \mu$ and $6.96 \mu$ and a third smaller maximum at $6.70 \mu$. For aragonite there are two bands, one simple band with maximum at $6.65 \mu$ and one complex band of two maxima at $6.46 \mu$ and $6.70 \mu$ respectively. In the second region the bands for calcite and aragonite are very similar but shifted in position as follows : calcite I I. $30 \mu$, aragonite II. $55 \mu$. But the bands for the two crystals coincide in the third region.

Calcite - extraordinary ray
$14.06 \mu, \quad$ ordinary ray $\mathrm{I} 4.17 \mu$. Aragonite $-c$

$$
\text { I } 4.06 \mu, \quad a \text { and } b \quad \text { I } 4.17 \mu \text {. }
$$

3. The first bands occur only in the ordinary ray for calcite and the $a$ and $b$ directions for aragonite. For both crystals the second band is present only in the direction of the acute bisectrix. The shifted band at $14.17 \mu$ occurs only in the obtuse bisectrix for both crystals.

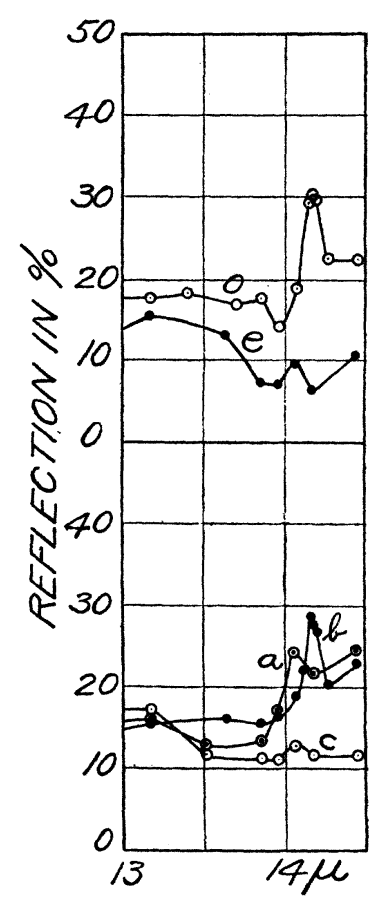

Figs. 12-13. Reflection from aragonite and calcite. $a$, vibrations $\| a$ axis; $b$, vibrations $\| b$ axis ; $c$, vibrations $\| c$ axis; $o$, ordinary ray ; $e$, extraordinary ray. $2.46 \mu$ absorption characteristics in a biaxial vibration and independent of the ray direction through the crystal.

5. At $14.06 \mu$ the reflection bands for the acute bisectrix in both substances have corresponding absorption bands which are very shallow. In the obtuse bisectrix the absorption band for calcite falls at $14.06 \mu$ while the corresponding reflection band is shifted to I $4.17 \mu$. 
[VoL. XXVIII.

6. The characteristic bands may be determined more advantageously by reflection than absorption, first on account of the difficulty in working with sections sufficiently thin for sharp bands, secondly on account of small errors which may result from impurity of the substance. A small per cent. of any impurity of high absorbing power in sections of appreciable thickness, may vitiate the results to a considerable degree. By the method of reflection such an error would be inappreciable.

7. From the physical similarity of the anhydrous carbonates of the calcite and aragonite groups, it may be assumed with safety that any characteristic of one of the members will apply equally well to all of the carbonates of that group. Accordingly the characteristic absorption and reflection bands at $6.6 \mu$ are not present in the acute bisectrix, at I I. $5 \mu$ they occur only in the acute bisectrix, for all of the anhydrous carbonates. The bands at $14 \mu$ are present in the absorption and reflection curves of each of the principal directions of vibration.

The present investigation has been carried out during the past year under the direction of Professors Nichols and Merritt, whom the author wishes to thank most heartily for the many facilities placed at his disposal and for their never-failing interest in the progress of the work. $\mathrm{He}$ is also much indebted to Dr. W. W. Coblentz, of the Bureau of Standards, for advice based upon his extended experience in radiometric work, to Professor Shearer for many useful suggestions, and to Professor Gill, of the Department of Mineralogy, for kindly advice in mineralogical matters, and for his friendly interest shown in other ways.

Cornell University, August, 1908. 